\title{
COMUNITARISMO Y NACIONALISMO
}

i partimos de la idea común de que una filosofía política es esencialmente un conjunto de argumentos morales sobre la justificación tanto de las instituciones como de la acción política podemos acotar inicialmente el tema así: ¿Tiene el nacionalismo un mejor punto de apoyo en los argumentos morales del comunitarismo que en los de la filosofía moral liberal? Es decir: ¿Es el comunitarismo un conjunto de argumentos morales particularmente idóneo para justificar ese conjunto de convicciones, actitudes e ideas políticas que llamamos nacionalismo? ¿Hay una buena sinfonía de base entre el nacionalismo como proyecto político y el comunitarismo como teoría moral? Si tuviéramos que dar una respuesta afirmativa a estos interrogantes tendríamos que concluir también que el nacionalismo tiene una dificultad considerable para configurarse como una actitud política que diera satisfacción a las exigencias morales del liberalismo, o, para formularlo en la otra dirección, que la filosofía moral liberal no suministra puntos de apoyo idóneos para justificar el nacionalismo. Las reflexiones que siguen tratan de suministrar algunos datos para orientarse en estas cuestiones. En cierto sentido puede muy bien decirse que se inspiran en puntos de partida que pueden llamarse, en la acepción precisa que tiene hoy esta palabra en la filosofía moral, «liberales» y que personifican de una manera especialmente luminosa trayectorias vitales e intelectuales como la de Ernesto Garzón Valdés, alguien que no acepta los argumentos del comunitarismo y que, desde luego no es nada nacionalista, lo cual no le impide, por cierto, sentir un genuino dolor por los problemas de su vieja y lejana patria.

La dicotomía entre comunitarios y liberales, o entre comunitaristas e individualistas, que hoy nos ocupa tanto en la teoría moral, 
puede que no sea sino un nuevo reflujo de contraposiciones muy hondas que parecen convivir en la historia del pensamiento y de la cultura y hacen su aparición periódicamente en los distintos ámbitos de la reflexión social, política y científica. Creo que será útil recordar algunas de esas dicotomías y tratar de situar nuestro tema en su marco para ir, poco a poco, definiendo las coordenadas en que se inscriben los distintos rasgos del comunitarismo. Quizás así podríamos también intentar una presentación general de la naturaleza de esta «nueva» teoría moral. La primera de esas grandes dicotomías podría ser la dicotomía entre naturaleza y cultura. Dentro de ella me parece que el comunitarismo se dejaría situar predominantemente en el ámbito de la naturaleza más que en el de la cultura, puesto que, al menos en sus versiones más rotundas pretende hablarnos de realidades que tienden a estar más allá de la inevitable artificiosidad de la cultura, es decir de realidades humanas naturales o muy parecidas a las naturales, en el sentido de que son imposibles de «construir» deliberadamente. Paradójicamente otra de las grandes dicotomías del pensamiento, la dicotomía entre razón e historia vería cómo el comunitarismo tendería a situarse más bien del lado de la historia que del lado de la razón. No es una simple construcción o reconstrucción racional lo que permite dar cuenta de las realidades de que habla el comunitarismo, sino una reconstrucción histórica: sin historia no puede darse la «comunidad» de los comunitaristas. La paradoja de que en la primera dicotomía se sitúe en el ámbito de lo natural y en la segunda dicotomía en el ámbito de lo histórico se produce porque en el primer caso trata de eludir la inevitable componente de «artificio» que tiene toda cultura frente a la naturaleza, mientras que en el segundo caso lo que trata de evitar es lo que tiene de «constructo» lógico, deliberado, aquello que se presenta como racional, frente a lo que tiene de «cronológico», heredado, tradicional lo histórico. La paradoja, a mi juicio, sólo es aparente, puesto que ambas opciones se sustentan en criterios cuyo paralelismo pudiera enunciarse así: postergar todo lo que de artificial tiene lo construido racionalmente en favor de lo que tiene de natural aquello que ha surgido anónimamente en la historia. La tercera gran dicotomía cultural, consabido eje de coordenadas de la teoría social y política, es la dicotomía entre individuo y sociedad. No es necesario decir que en esta dicotomía parece evidente que el comunitarismo se ubica claramente, aunque con diferentes grados de intensidad según las propuestas, del lado de la sociedad. Para el comunitarismo la realidad humana primaria y original es la sociedad y no el individuo.

Todas estas dicotomías, y algunas más, tienen también su correlato en la teoría política. Es relativamente sencillo presentar, por ejemplo de la mano de Bobbio (Bobbio, 1985, págs. 92 y ss.), un par 
opuesto de modelos teóricos con los que la filosofía política ha tratado de dar cuenta de su objeto de estudio. De acuerdo con el primer modelo, llamado por Bobbio «modelo hobbesiano», el espacio político, es decir, la politeia, la respublica, la sociedad política o el estado, como se prefiera, es un producto cultural, una construcción artificial de la razón, lógica y deliberada, llevada a cabo por individuos preexistentes, libres e iguales por naturaleza, mediante un consentimiento que crea o constituye esa realidad nueva llamada sociedad política o estado. De acuerdo con el segundo modelo, llamado por Bobbio «modelo aristotélico», la comunidad política es una herencia, producto de un desarrollo histórico y cronológico desde formas naturales de sociabilidad (por ejemplo, la familia) hacia formas más complejas, pero nunca deliberadamente creadas por el consentimiento o la razón, sino forjadas en la tradición y la historia, y dentro de las cuales y de conformidad con roles y dependencias preestablecidas, «habitan», por así decirlo, los individuos.

En realidad, la ontología social que se transparente en uno y otro modelo vuelve a repetirse, como un reflejo más, a lo largo de la teorización sociológica de fin de siglo pasado. Me refiero a esa distinción entre «comunidad»y «asociación» que formula Tönnies, a la paralela distinción entre solidaridad «mecánica» y «orgánica» de Durkheim, o a la teoría de los grupos «primarios» y «secundarios» de Cooley. Como se sabe, para Tönnies, la comunidad es un organismo vivo, con vida orgánica y real, basado en la convivencia íntima, privada, excluyente, con vínculos de sentimiento y relaciones muy inmediatas, que expresa unidad y reciprocidad, se sustenta en fines y objetivos compartidos y tiene una gran fuerza como criterio de identificación colectiva. Su germen, una vez más, es la familia, y dentro de ella la relación materno-filial. Tönnies subraya que es algo que tiene valor en sí mismo, hasta el punto de que hablar de una Gemeinschaft «mala» resulta ser una contradicción en los términos. Por su parte, la sociedad o asociación se constituye mediante estructuras imaginarias y mecánicas, urdidas, construidas, diseñadas artificialmente, no vitalmente, producto de la voluntad, de la razón, del contrato que establece normas externas que se programan para constituir el grupo. No tiene ningún poder identificatorio ni valor alguno en sí y como tal. Es, simplemente, una realidad creada a partir de normas, como una escritura notarial crea una sociedad mercantil, establece las condiciones para ser socio, los órganos de gobierno de la sociedad y los derechos y deberes de cada uno (cfr. Tönnies, 1979).

Naturalmente el comunitarismo se ubica como es lógico mucho más cerca del «modelo aristotélico»y, por supuesto, de la teoría sociológica de la «comunidad», que del «modelo hobbesiano» y de la teoría sociológica de la asociación con vínculos de solidaridad 
meramente mecánicos. El éxito de la filosofía moral comunitaria en este fin de siglo no sería así, sino un reflujo más de uno de los términos de esa antigua dicotomía, Incluso cabría hacer una especie de conjetura histórico-sociológica: Igual que, como se ha dicho, la oleada industrializadora generó la añoranza por la idea de comunidad agraria a finales del siglo XIX, la gran oleada tecnológica actual, la universalización de los procesos económicos, y la ajeneidad y atomización del ser humano en sociedades ultradesarrolladas, producen también hoy la vuelta hacia comunidades intensas y cercanas que sustenten la posibilidad de relaciones vitales inmediatas para los seres humanos.

II

Vamos a considerar ahora algunos aspectos generales del comunitarismo y, en particular, algunas dimensiones o implicaciones políticas del mismo. Para empezar parece cuestión pacífica entre los glosadores del comunitarismo que el enfoque que éste usa para justificar los valores morales es un tipo de enfoque que Walzer ha denominado «justificación local», y que «asume que en el fondo la gente comparte una vida común», y que «podemos llegar a acuerdos sobre lo bueno a través de una mejor interpretación de nuestros compromisos» (Thigpen and Downing, 1989, pág. 536). Los liberales, por el contrario, apelan a la llamada «justificación cosmopolita» que necesita puntos de partida que superen los límites contextuales en que se desarrolla el debate moral y la socialización empírica de los agentes. Como ha visto bien Carlos Thiebaut (Thiebaut, 1992) esta tensión entre términos teóricos como universalidad y particularidad desborda su carácter puramente epistémico para manifestarse como tensión política en la configuración de las sociedades occidentales. De nuevo una gran dicotomía, o quizás simplemente otra versión de una de las grandes dicotomías que antes veíamos, y que ahora aparece con una dimensión claramente política: en este caso entre el particularismo político y el cosmopolitismo de una política universalista. La teoría comunitarista, pues, se inclina por una metodología que lleva implícita la idea de que la moralidad -y por tanto también la moralidad que ha de subyacer a las instituciones políticas- no puede ser concebida en términos universales, sino sólo en términos de ámbitos vivenciales concretos. Incidentalmente recordaré tan solo lo que esto supone en términos de rechazo de pautas y criterios morales que, como los derechos humanos, muestran una clara vocación universalista (Sobre ello, cfr. Ruiz Miguel, 1992).

En segundo lugar, y conectado con lo anterior, está, naturalmente, el fundamental compromiso ontológico que supone la noción fuerte 
de «comunidad». Salir con bien de la tarea de ofrecer un concepto de «comunidad» no es nada fácil. Cualquier diccionario de Sociología, Política o Filosofía que utilicen nos advierte de esto. Por ejemplo: «El término "comunidad" es uno de los más vagos y escurridizos de la sociología, y de momento carece, en gran medida, de sentido específico» (Abercrombie y otros, 1994). Tan es así que ya ha sido incluido en la nómina de los llamados conceptos esencialmente contestados, es decir, aquellos conceptos cuyos criterios de uso son insaciables y permanentemente debatidos (Plant, R. 1978). No es fácil, por ello, encontrar entre los comunitaristas definiciones muy explícitas. En el «Glosario de algunos conceptos clave» del conocido libro de Robert N. Bellah (Bellah y otros, 1989) encontramos una tan arbitraria como otra cualquiera, pero ilustrativa de algunos puntos: «un grupo de personas que dependen socialmente una de otras, que participan juntas en los debates y en la toma de decisiones y que comparten ciertas prácticas que a la vez definen la comunidad y son alimentadas por ella». En cuanto al concepto de práctica los autores en cuestión, tras las huellas de MacIntyre, afirman que no son actividades puramente instrumentales y que no se caracterizan simplemente por su aspecto repetitivo, sino que «son éticamente buenas de por sí». No necesito recordarles a este respecto el concepto de práctica del propio MacIntyre: «...cualquier forma coherente y compleja de actividad humana cooperativa establecida socialmente, y a través de la cual los bienes internos a esa forma de actividad son realizados en el curso de los intentos para alcanzar aquellos standards de excelencia que son apropiados a, y parcialmente definitorios de, esa forma de actividad, con el resultado de que los poderes humanos para lograr la excelencia y las concepciones humanas de los fines y bienes implicados, son sistemáticamente extendidas» (MacIntyre, A. 1982, pág. 187). No se trata, pues, cuando hablamos de esas prácticas sociales en que consiste, y que definen a la «comunidad» de la noción común de prácticas sociales, sino de algo que lleva ya en sí, como un lastre significativo, aspectos morales normativos. La idea de comunidad no se separa por ello tampoco ahora de la original idea aristotélica de que «...toda comunidad está constituida en vista de algún bien...» (Política, libro I, 1252 a), ni de la de Tönnies de que «...hablar de mala Gemeinschaft viola el significado del término» (Tönnies, 1979, pág. 28). La comunidad es, por tanto, una realidad en la que se encarna necesariamente un bien en sentido moral.

Todas estas consideraciones ya sabidas vienen a cuento porque tienen una dimensión política innegable. Esa dimensión política puede ponerse de manifiesto haciendo una referencia indirecta a la teoría de la legitimidad, o mejor, a la teoría de la obligación política, sugiriendo que con respecto a la obligación política los comunitaristas, 
casi inadvertidamente, proceden a trasladar la carga de la prueba, y esto tiene algunas importantes consecuencias. Trataré de explicarme. De acuerdo con los presupuestos de la filosofía liberal, el individuo dotado de derechos morales es un dato previo a la construcción y diseño de las instituciones políticas, y éstas adquieren su justificación en la medida en que son un vehículo para la garantía de esos derechos morales individuales. Como quiera que la mera existencia de esas instituciones, con su coacción organizada, limita ciertos derechos individuales, son esas instituciones las que tienen la carga de la prueba de su propia legitimidad, es decir, son ellas las que tienen que justificar su propia existencia. Esto es extremadamente importante porque esa necesidad incesante de justificarse a sí mismas es lo que explica la paulatina y cada vez más profunda evolución liberal de las formas políticas de gobierno y las permanentes exigencias de articular las instituciones y los poderes conforme a pautas de democracia e igualdad. Este proceso de perpetua autocrítica es inconcebible en el comunitarismo, porque en él el dato previo que preexiste al debate ético-político es precisamente la existencia de la comunidad dotada de cierto valor intrínseco. Y ello determina, en primer lugar, que sea el ciudadano el que soporte la carga de la prueba, es decir, el que tenga que justificar sus reticencias ante la obligación de obedecer las normas, el que, en definitiva, tenga que responder a la pregunta por la obligación política. Las instituciones, en la medida en que encarnan ese bien intrínseco, tienen un derecho «prima facie» a exigir lealtad. A pesar de todos sus matices la posición de MacIntyre implica esto: el patriotismo supone que al menos algunos de los proyectos y prácticas de mi nación «deben estar permanentemente exentos de crítica» (MacIntyre, 1984, pág. 13). Y de esto quedan también en alguna medida exentas las instituciones políticas que dan forma a mi nación, contra la propuesta que hace el propio MacIntyre, porque no sirve distinguir entre la política, que puede ser criticada, y la nación, que no puede serlo. Si la nación es un conjunto de proyectos y prácticas tiene necesariamente que tener una articulación política e institucional (de lo contrario estamos fabulando una entidad perfectamente imaginaria o mágica). En consecuencia, el ciudadano tiene una obligación «prima facie» de carácter político y si quiere ignorarla tiene que argumentar él. Y si seguimos con MacIntyre, sospecho que argumentará en vano, porque la patria es también un conjunto de tradiciones, y las tradiciones tienen incorporada una suerte de racionalidad interna (la historia es racional). El ciudadano, por tanto, tiene también un límite para acudir al arsenal donde están depositadas las razones. La comunidad, tengo la impresión, está blindada contra toda crítica. 
Me parece que estas reflexiones no se aplican sólo al pensamiento, particularmente conservador, por así decirlo, de MacIntyre. Creo que están implícitas en cualquier modelo semejante, es decir, en cualquier modelo que atribuya un valor intrínseco a la mera existencia de la comunidad. Sandel, por ejemplo, no duda en afirmar que existen también esas lealtades y fidelidades fuertes (Sandel 1984, pág. 23), porque, obviamente, si el «yo» no puede ser concebido sin lastres, sin arraigos, esto va mucho más allá de una mera consideración epistémica: es esencialmente una cuestión práctica porque si niego a mi comunidad política me niego a mí mismo, si la traiciono me traiciono a mí mismo. No es simplemente que yo no me entienda a mí mismo como agente moral, como ciudadano: es mucho más que eso, es que al poner en cuestión mi comunidad moral o mi comunidad política me estoy poniendo en cuestión a mí mismo.

Mencionaba esto para enlazar con el siguiente tema que me interesa, que es aquel de la pertenencia, «membrecía» o, en términos más decididamente políticos, ciudadanía. Entre los comunitaristas, como es bien sabido, Sandel es particularmente importante a este respecto porque postula con una particular fuerza el carácter constitutivo de la comunidad tanto en términos de agente moral como en términos de ciudadanía política: «una comunidad tal que envolvería la identidad tanto como los intereses de los participantes, y así implicaría a sus miembros en una ciudadanía más profunda (through-going) que la que el yo desarraigado pueda conocer» (Sandel 1984, pág. 19). Es decir, que de la misma manera que el agente moral es «constituido» por la comunidad moral el ciudadano sería constituido por esa especie de comunidad moral que sería la politeya. Pues bien, al margen de las aporías en que desemboca esta «noción homogénea y no compleja de identidad y comunidad», y que han sido analizadas lúcidamente por Thiebaut (Thiebaut 1992, págs. 161 y ss.), mi consideración es otra. ¿Cómo es posible eludir la idea de que desde esta perspectiva teórica la moralidad conformaría una especie de geografía dentro de la cual ciertos sujetos pertenecen a un territorio y otros no? Si la comunidad nos constituye como agentes morales, nos adscribe nuestra condición moral, entonces los agentes humanos de una comunidad serán, por fuerza, moralmente distintos a los de otra comunidad. Y con ello se habrá establecido una clara distinción entre «nosotros» y «ellos». Esto no significa ni más ni menos que nuestro obrar moral respecto de los otros, de los «ellos», resulta modificado por las distintas pertenencias, lo que es o puede ser la base de discriminaciones e intolerancias (Cfr. Thiebaut, 1992, pág. 58). Contra esta amenaza quizás podría oponerse la teoría de Walzer sobre la distribución de la «membership», pero tengo que confesar que, al margen de algunos matices, y dicho en trazos un 
poco gruesos, tal idea no me parece, en el contexto teórico en que está expuesta, demasiado comprensible. ¿Qué significa que se pueda manipular, es decir, constituir por nuestra comprensión y fijar por nuestro trabajo y conversación (Walzer 1983, pág. 66), ese bien que es la «membership»? ¿Qué significa que una vez así definida podamos nosotros «distribuirla»? ¿Quiénes somos «nosotros»? Todo parece indicar que una serie de sujetos pueden adscribir la pertenencia o membrecía y con ello la identidad moral a otros sujetos. Esto en el pensamiento liberal es fácil de entender por lo que respecta al ámbito político (es la concesión arbitraria de ciudadanía por carta de naturaleza), pero en el lenguaje comunitario es imposible de concebir. Porque ¿cómo se puede ser miembro de una comunidad comunitaria «por decreto», por así decirlo? Si el depósito de identidad moral se encuentra en la comunidad, aceptar que unos sujetos puedan administrarlo deliberadamente de este modo resulta decididamente contradictorio. Porque si aceptamos que alguien sin vínculos, sin tradición, sin historia, sin parientes, sin cultura y sin «entendimientos compartidos» puede ser agraciado por algunos miembros de un grupo con la condición de «miembro»o «perteneciente», entonces ¿qué papel juega en todo esto la «comunidad»?

Vamos a pasar ahora a otro tema interesante. Aunque no se formulase explícitamente, antes se ha querido sugerir que la idea tan cara a MacIntyre, de que ha de fomentarse una virtud que consista en el hábito de desarrollar comportamientos leales y patrióticos hacia la comunidad, deja a un lado la cuestión peliaguda de si tal lealtad es debida cualquiera que sea el sistema político en el que se articula esa comunidad. El comunitarismo sería así indiferente respecto de cuestiones tan fundamentales políticamente como la de las formas de gobierno. Me parece que esto se complica más cuando vemos la dificultad que encuentran los comunitaristas para entroncar el sistema democrático en su teoría moral. Porque en efecto, para nuestra sorpresa, nos encontramos con que la mayoría de los comunitaristas que se confiesan demócratas parecen ser partidarios de un tipo de democracia muy especial, de moda en la teoría, pero completamente inédito en la realidad, y que se viene llamando «democracia participativa». Y ello se debe seguramente a que la ontología de la «comunidad» establece sobre los tipos de organización política aceptables fuertes condicionamientos. De acuerdo con esa ontología las instituciones políticas han de construirse sobre las «autocomprensiones compartidas» (Sandel), sobre la «vida común» basada en la persuasión y el argumento (Walzer), sobre la «común deliberación, la común decisión y la común acción» (Barber). La democracia «fuerte» de Barber, en contraste con la democracia «flaca» que conocemos, es un proceso participativo de conversación, es «el arte de 
envolver a los extraños en la conversación y estimular en ellos un parentesco artificial hecho a partes iguales de empatía, causa común y autointerés ilustrado» (Thigpen and Downing, 1987, págs. 645 y ss.). Y claro, resulta muy evidente que todas esas actividades que consisten en argumentarnos, persuadimos unos a otros, deliberar en común, y sentir empatía es algo sólo posible en relaciones humanas muy cercanas, es decir, en relaciones humanas no mediadas. Y aquí el comunitarismo pone de manifiesto que todavía sigue vinculado a la idea de comunidad como ámbito de convivencia íntima, cercana, con vínculos de sentimiento, relaciones muy inmediatas y fines y objetivos compartidos. Lo que significa que tal modelo de democracia no parece servir para comunidades políticos muy grandes, ni para sociedades multiculturales y heterogéneas. E incluso cabría dudar de que sirva para pequeñas comunidades políticas, porque tanto deliberar, persuadir y parlamentar tiene un coste personal en tiempo que pocos socios estarían dispuestos a pagar, y que en el fondo casi nadie consideraría necesario porque con sentimientos tan cercanos, fines tan compartidos y una autocomprensión común tan profunda ¿de qué hay que discutir? Cualquier comunero, por turno, podría hacer lo que todo el mundo sabe que se tiene que hacer.

Voy, por último, a hacer una pequeña excursión por uno de los temas en los que la crítica comunitaria al liberalismo parece más potente. Me refiero a la imposibilidad del liberalismo individualista y universalista para dar cuenta de nuestros deberes morales especiales hacia los seres más cercanos. Me interesa porque creo que sobre el molde de esos deberes se quiere construir la idea de lealtad política, y eso tiene implicaciones que no pueden ignorarse. Dworkin describe así la situación: «creemos que tenemos particulares responsabilidades hacia aquellos con quienes tenemos especiales relaciones: nosotros mismos, nuestra familia, amigos y colegas. Gastamos más tiempo y más recursos en ellos que en los extraños, y pensamos que eso es lo correcto» (Dworkin 1992, págs. 221-222). Los comunitaristas se muestran particularmente de acuerdo con este esquema, hasta el punto de que toda su teoría moral gira en torno a él, y de acuerdo con él quisieran también diseñar el vínculo político. En consecuencia, afirmarían que también hemos de tener hacia nuestros conciudadanos un «special concern» que no tenemos con los extranjeros. Desde luego, el característico universalismo de la filosofía liberal no tiene una fácil respuesta para esto: $\mathrm{Si}$ todos los seres humanos son moralmente iguales no parece haber ninguna razón para que mis obligaciones con respecto a los cercanos sean más intensas que para los demás. Sólo lo que Walzer ha llamado «universalismo reiterativo» (Walzer 1990, pág. 531) sería capaz de dar cuenta de ello: ese «special concern» hacia mis hijos, mis amigos, 
mis compañeros y mis conciudadanos sería el producto de los actos de especialización moral en el marco del universalismo reiterativo. Dejando a un lado la idea del universalismo reiterativo, que sería digna de análisis, lo que me interesa es esa sugerencia de que puede darse una «especialización moral» $\mathrm{o}$, como ha afirmado alguien muy poco comunitarista, que hay una «división del trabajo moral» (Henry Shue 1988, pág. 687). Por ahí me parece que habría que explorar la respuesta a la crítica comunitaria, pero no voy a hacerlo. Lo único que quiero es llamar la atención sobre el hecho de que los comunitaristas establecen esa «especialización moral» sobre la base de relaciones afectivas: familia, amigos, compañeros... hasta incluir conciudadanos, como si la relación de conciudadanía tuviera un obvio componente sentimental. Y esto me inspira dos reflexiones: en primer lugar, que es posible que esa concepción de la especialización moral se sustente en una confusión entre la justificación de los deberes y la motivación para cumplirlos (la sempiterna confusión entre razones en sentido justificatorio - razones en sentido estricto - y razones en sentido explicativo - motivos). Y en segundo lugar, que esa concepción de la especialización moral, para hablar en términos de Hume, opta por la pasión en lugar de por la razón, es decir, opta por el sentimiento frente a la racionalidad. Y eso es sumamente problemático porque, como ya nos advirtió el propio Hume, las pasiones se debilitan con la distancia y con el tiempo, de forma tal que la mayor fuerza de nuestros deberes morales sería para los cercanos en el espacio y en el tiempo. La cohesión moral entre «nosotros» sería muy intensa: nuestros deberes para con las gentes exteriores, más débiles, y con personas remotas en el espacio o el tiempo, inexistentes. Y, claro está, este tipo de consideraciones son muy relevantes para calibrar qué lógica interna puede presidir la posición comunitarista en materias como la solidaridad internacional o las relaciones entre comunidades o pueblos distintos.

\section{III}

Vamos ahora a realizar una breve indagación por entre la trama del ideario nacionalista. Para hacerlo vamos a partir de una distinción que he hecho en algún trabajo anterior entre «nación-organización» y «nación-alma». No estoy seguro de que ésta sea la más feliz de las terminologías pero creo que su sentido está razonablemente claro. La «nación-organización», que corresponde a lo que hoy llamamos Estados nacionales, es una organización político-administrativa definida, con un sistema jurídico con criterios de identidad razonablemente claros y un ámbito de validez de sus normas acotado en 
lo personal y en lo territorial. Al margen de la parafernalia sentimental y cultural que se pretenda adherir a ella, su núcleo fundamental es la estructural institucional de carácter jurídico, político y administrativo en que se sustenta. Respecto de esa idea de nación cabe hacer dos consideraciones. En primer lugar, en ella el ámbito territorial está racionalizado. En segundo lugar, los problemas de «membrecía» están resueltos con arreglo a criterios razonablemente claros y explícitos. Me explico. Si los teóricos de este modelo de nación tienen razón en sus análisis entonces este concepto lleva consigo la idea subyacente de que las fronteras son algo que puede ser determinado por «razones» instrumentales, y en último término, algo que puede ser «establecido» (como se hizo en África durante el XIX y el XX) en función de criterios prudenciales o convencionales. Naturalmente todos saben que las fronteras de las viejas Naciones-Estado (como también son llamadas) son el producto de azares históricos, circunstancias arbitrarias, contingencias imprevisibles, etc..., pero en la medida que saben eso, saben también que no son ni naturales ni sagradas, es decir, que son un potencial tema de disputa y desacuerdo, y por tanto también de negociación y arreglo. Hoy eso es, por lo que respecta a ese tipo de naciones, materia asumida. Si hay desacuerdo fronterizo se establecen sistemas de arbitraje (el Papa, el Secretario de las Naciones Unidas, etc...) y se salda el conflicto restableciendo mediante «razones» el equilibrio de intereses. Lo que me interesa destacar de esto es precisamente esa posibilidad implícita de dibujar el mapa de una organización político-administrativa en base a razones intersubjetivamente compartibles. La otra consideración se refería a la pertenencia del sujeto a la nación, a la «ciudadanía» o la «membrecía» (para usar otra vez este americanismo que parece inevitable). Y por lo que respecta a esto sucede lo mismo. Los criterios para obtener la condición de miembro de esas comunidades políticas pueden ser modificados, diseñados y manipulados en base a argumentos inteligibles y pueden ser establecidos en normas jurídicas. Por cierto que tales criterios pueden parecer arbitrarios para quien tenga una idea demasiado «sanguínea» de lo que es la nacionalidad, pero vistos desde otra perspectiva pueden obedecer a razones mucho más «racionales», por así decirlo, que el llamado «ius sanguinis». Las concesiones de nacionalidad por carta de naturaleza son un buen ejemplo de ello. Lo que nos indican es que la adscripción de ciudadanía puede estar gobernada por la racionalidad, es decir, puede estar basada en «razones» y razones de todo tipo: prudenciales o morales (económicas, políticas, deportivas, humanitarias, etc...).

Pero pasemos ahora a lo que llamaba la «nación-alma». Es terminología deudora de una tradición larga. Es el equivalente a la 
«nación-genio». La caracterización es conocida, aunque difícil de sentar en el lenguaje: grupo humano como alma colectiva, totalidad inclusiva, basada en tradiciones y con hondas raíces en un pasado, que se constituye por vínculos «naturales» orgánicos y expresa una comunidad viva de lengua, raza y cultura (Delannoi 1993, pág. 45). Frente a la pre-existencia, que antes hemos visto, de algún tipo de dominación política, realidad económica, u organización administrativa, aquí lo que se afirma que preexiste a todo es esa realidad denominada «pueblo». Algo muy determinado, o al menos usualmente definido, mediante una metáfora del parentesco, en la que el ius sanguinis y los lazos afectivos son muy importantes, que se diseña mediante una apelación constante a la familia. Para esta concepción de la nación hay una suerte de división natural de la especie humana por razón de su raza, de su lengua y de su hábitat vital, y el resultado de esa división natural son las naciones. Esta articulación inevitable de la especie humana en «naciones» se manifiesta en la historia y en la tradición. Para los nacionalistas de esta especie, la historia y la tradición son fuentes de identificación y origen de pautas de organización social. Los partidarios de este tipo de entidad nacional descansan, en efecto, muy especialmente en la historia. En ella y en la lengua, la cultura y la literatura localista. Con cierta frecuencia también en la religión. A la hora de definir todo eso se recurre mucho más a la voluntad colectiva, a los fenómenos plebiscitarios tácitos, que a la racionalización de la organización y sus fines. Una amalgama de todas esas cosas, que no en vano se ha tachado siempre de confusa, constituye la nación en este sentido. Esa confusión se produce porque mantiene que, al margen de cualquier estructura institucional dada, las naciones son conocidas por ciertas características que pueden ser descubiertas y establecidas, y se ha dicho con toda razón que no hay manera de validar esta proposición (Birch 1993, pág. 16). Pues bien, a pesar de ello esa característica amalgama que mezcla en distintas dosis raza, lengua, sangre y linaje, historia y tradición, religión y una mística y cotidiana «voluntad de ser» se confabula con demasiada frecuencia para hacer creer a muchos que hay ahí una unidad humana innegable y que esa unidad humana tiene una especie de derecho natural a desarrollar una dimensión política institucional. Esto es el fenómeno del nacionalismo. Cuando este fenómeno encuentra un contexto histórico propicio se impone culturalmente a los demás; cuando se despierta en un ambiente hostil se desarrolla, en palabras de Javier de Lucas, como «un proceso reactivo y en gran medida victimista» (Lucas 1992).

Esta peculiar manera de concebir las entidades políticas más importantes va acompañada también de una peculiar visión de la idea de frontera y de la idea de pertenencia o membrecía. Respecto a 
lo primero hay una especie de creencia en una cierta relación mística o natural entre una unidad humana y un territorio. Un lazo o vinculación que, cualquiera que sea su naturaleza: histórica, natural, etc... no puede ser puesto en cuestión sin poner en cuestión la naturaleza misma de ese todo social humano. Ésta es la teoría, tan difundida en tiempos, de las fronteras naturales. Los Pirineos, los Alpes y el Rhin son las fronteras naturales de Francia. Una franjita en el cercano oriente es el territorio natural de la patria judía. Y así sucesivamente. Como la realidad traiciona sistemáticamente este tipo de aseveraciones y las fronteras muestran todos los días su carácter arbitrario y convencional, entonces se utiliza la idea de las «fronteras interiores» que son eso lazos invisibles de que hablaba por ejemplo Herder al referirse a la vinculación de la lengua. Es decir, que ni aquellas fronteras naturales pueden ser concebidas como objeto de negociación, racionalización o regateo, ni, y esta es la segunda consideración, la idea de membrecía o pertenencia que dibujan las llamadas «fronteras interiores» puede ser objeto de decisión voluntaria.

Pues bien, eran sólo estas dos consideraciones lo que ahora me importaba hacer: Parece formar parte de la lógica interna de este modo de pensar que el ámbito de validez espacial de las normas de la sociedad política, es decir, el ámbito de la organización político-administrativa, está en gran medida dado de antemano y no es, por tanto, susceptible de ser diseñado en función de razones de uno u otro tipo. En segundo lugar, la condición de miembro o los criterios subjetivos de pertenencia a la nación tampoco pueden ser sometidos a una argumentación basada en razones. Si hay «razones» para la demarcación fronteriza y «razones» para la membrecía, son ambas «razones» que nos vienen impuestas desde la naturaleza o la historia, y por tanto son «razones» frente a las que poco podemos nosotros hoy «razonar».

Todo este mosaico de convicciones dibujan la idea de nación-alma en el molde de las llamadas características «étnicas». Y en consecuencia establecen una relación intensa entre nacionalismo y etnicidad. En efecto, la reciente publicación de un libro que trata de ver le nacionalismo desde la perspectiva de la antropología (una de las pocas perspectivas desde las que no se había examinado exhaustivamente) ha puesto de manifiesto de forma que a mí me parece muy contundente que el nacionalismo lleva consigo necesariamente componentes de «etnicidad» (Eriksen, 1993). La etnicidad es la conciencia viva en un grupo de que se dan diferencias culturales respecto de los otros grupos con que se relaciona, tales que permiten la clasificación sustantiva entre «nosotros» y «ellos», crean estereotipos, son la base de juicios fundamentalmente «adscriptivos» (no descriptivos) y demandan una organización social de la «distintividad cultural». Pues 
bien, creo que puede afirmarse que la idea de nación-organización no se basa necesariamente en una ideología que incorpore la «etnicidad», mientras que la idea de nación-alma no es más que la aplicación al ámbito de la política de una idea intensa, de «etnicidad», una idea que, según Eriksen, establece una relación definida entre «etnicidad» y «Estado». Por eso creo que el nacionalismo en sentido estricto, y sobre todo en sentido agresivo, deriva mucho más de la idea étnica de una comunidad con alma que de la artificiosa, aunque también estúpida y peligrosa a veces, creación cultural de unos arquetipos destinados a intensificar la cohesión social en una realidad colectiva ya dada.

\section{IV}

En su excelente libro sobre comunitarismo Thiebaut advierte de que «el carácter de la crítica comunitarista democrática norteamericana no es fácilmente transportable a este lado del atlántico, pues el debate se transforma aquí en el debate del nacionalismo y del cosmopolitismo» (Thiebaut 1992, pág. 146). Aun aceptando lo que de exigencia de precisión y rigor tiene esa advertencia, tengo sin embargo que decir que esa desviación del debate comunitario hacia el problema del nacionalismo es extremadamente fácil de explicar: la sintonía entre comunitarismo y nacionalismo es demasiado evidente como para que pueda extrañar. El comunitarismo es un tipo de teoría moral que suministra al nacionalismo argumentos que le son muy queridos. Incluso si empezamos por lo que parecería más disparatado, como es la relación mágica entre un grupo humano y un territorio, podemos, por ejemplo, leer en Walzer la siguiente afirmación: «Las naciones buscan países porque en algún sentido profundo ya tienen países: el vínculo entre la gente y la tierra es un rasgo crucial de la identidad nacional» (Walzer 1983, págs. 80-81). Es ese misterioso «vínculo profundo» de que habla Walzer con sentido aprobatorio lo que hemos visto en el famoso problema de las fronteras naturales. En segundo lugar la idea de comunidad preexistente como bien en sí, superior a la peripecia moral de sus componentes individuales y cuyos supuestos fundamentales han de mantenerse al margen la crítica se compadece perfectamente con la idea nacionalista de «pueblo» como entidad orgánica con valor moral, con el sacrificio característico de los individuos al destino nacional y con la tendencia nacionalista a sacralizar a la patria y su historia acríticamente, y fomentar la lealtad hacia la misma al margen de la forma política que su organización adopte. El nacionalismo, se ha dicho muchas veces, no tiene credo político. En tercer lugar, el comunita- 
rismo alienta el concepto natural, histórico o cultural de «membership» frente al concepto racional y deliberado de «ciudadanía», al afirmar que la comunidad, entendida en confusos términos histórico-culturales, tiene una dimensión constitutiva del agente moral y político. En cuarto lugar, el comunitarismo mantiene que las pautas de comportamiento social y político tienen una mejor justificación local, interna la cultura, intrahistórica, que universal, transcultural y suprahistórica. Como afirmaba antes Thiebaut, el comunitarismo en este sentido es un perfecto correlato del nacionalismo particularista. En quinto lugar, el comunitarismo ofrece una plataforma teórica óptima para apoyar la distinción entre «nosotros» $\mathrm{y}$ «ellos», «insiders» y «outsiders», y con ello se inscribe en el grupo de aquellas teorías que usan entre sus ingredientes la noción técnica de «etnicidad», algo que es, según recientes investigaciones, arquetípico del nacionalismo y de su distinción político-moral básica entre «nacional» y «extranjero». Y, por último, ese género de cohesión moral de carácter sentimental sobre el que se quiere edificar la comunidad como colectivo de seres «cercanos» encajaría perfectamente como descripción del tipo de cohesión emocional que trata de inducir el nacionalismo entre los ciudadanos. Y se sustenta en el mismo error: articular la división necesaria del trabajo moral, la especialización moral, en base a las motivaciones sentimentales del obrar en lugar de sobre la justificación del deber.

Todas estas razones nos deben llevar, pues, a concluir que comunitarismo y nacionalismo tienen un claro parecido de familia. $\mathrm{Y}$ en la medida en que el nacionalismo puede ser comprendido y justificado como algo que deriva de la lógica interna de una filosofía moral de tipo comunitarista, entonces algunos evidentes corolarios del nacionalismo y algunas de las experiencias históricas que ha desencadenado podrían ser objeto de esa comprensión y de esa justificación. Los comunitaristas nos tienen todavía que decir si, como parece, esto es así. 


\section{REFERENCIAS}

Abercrombie y otros, Diccionario de Sociología. Ed. Tecnos. 1994.

Avineri, S. y De-Shalit, A., Communitarianism and Individualism. Oxford University Press. Oxford. 1992.

Bellah, R. N. y otros, Hábitos del Corazón. Alianza Universidad. Madrid. 1989.

Birch, A., The Concepts and Theories of Modern Democracy. Routledge. New York. 1993.

Delannoi, G. y Taguieff, P. A., Teorías del Nacionalismo. Paidós. Barcelona. 1993.

Dworkin, R., Liberal Community, en Avineri y De-Salit. 1992.

Eriksen, M., Ethnicity and Nationalism. Pluto Press. London, Boulder. 1993.

Lucas, Javier de, Europa: ¿Convivir con la Diferencia? Ed. Tecnos. Madrid, 1992.

Plant, R., «Community: Concept, Conception and Ideology». Politics and Society 8, 1978.

Ruiz Miguel, A., Derechos Humanos y Comunitarismo. Aproximación a un debate. Doxa n. ${ }^{\circ} 12$. 1992.

Shue, H., Mediating Duties, Ethics, 98, julio 1988.

Thiebaut, C., Los Limites de la Comunidad. Centro de Estudios Constitucionales. Madrid. 1992.

Thigpen and Downing, Liberalism and the Communitarian Critique, American Journal of Political Science, vol. 31, n. ${ }^{\circ} 3.1987$.

- Liberal and Communitarian Approaches to Justification. The Review of Politics, 1989, vol. 51.

Walzer, M., Membership, fragmentos de Speheres of Justice 1983, ahora en Avineri y De-Shalit 1992.

- National and Universe. The Tanner Lectures on Human Values, vol. XI. 1990. 